\title{
A Social Network Analysis of Engineering Faculty Connections: Their Impact on Faculty Student-Centered Attitudes and Practices ${ }^{\dagger}$
}

\author{
James A. Middleton*(D), Stephen Krause, Eugene Judson $\mathbb{D}^{\mathrm{D}}$, Lydia Ross, Robert Culbertson, Keith D. Hjelmstad $\mathbb{D}_{\text {, }}$ \\ Kara L. Hjelmstad and Ying-Chih Chen (D)
}

\section{check for}

updates

Citation: Middleton, J.A.; Krause, S.; Judson, E.; Ross, L.; Culbertson, R.; Hjelmstad, K.D.; Hjelmstad, K.L.; Chen, Y.-C. A Social Network Analysis of Engineering Faculty Connections: Their Impact on Faculty Student-Centered Attitudes and Practices. Educ. Sci. 2022, 12, 108 https://doi.org/10.3390/ educsci12020108

Academic Editor: James Albright

Received: 16 December 2021

Accepted: 31 January 2022

Published: 4 February 2022

Publisher's Note: MDPI stays neutral with regard to jurisdictional claims in published maps and institutional affiliations.

Copyright: (C) 2022 by the authors. Licensee MDPI, Basel, Switzerland. This article is an open access article distributed under the terms and conditions of the Creative Commons Attribution (CC BY) license (https:// creativecommons.org/licenses/by/ $4.0 /)$.
Ira A. Fulton Schools of Engineering, Mary Lou Fulton Teachers' College, Arizona State University, Tempe, AZ 85287, USA; Stephen.Krause@asu.edu (S.K.); Eugene.Judson@asu.edu (E.J.); 1lross@asu.edu (L.R.); robert.culbertson@asu.edu (R.C.); keith.hjelmstad@asu.edu (K.D.H.); kara.hjelmstad@asu.edu (K.L.H.); ychen495@asu.edu (Y.-C.C.)

* Correspondence: jimbo@asu.edu

+ Portions of this manuscript were presented at the 2016 ASEE Annual Conference, New Orleans, LA, USA, 26-29 June 2016.

\begin{abstract}
Connections among faculty who might be potential resources for diffusion of learnercentered practices across STEM departments were examined at a major research university. Specifically, the research assessed the impact of these connections on learner-centered beliefs among faculty and the implementation of learner-centered practices across the network. Participants were recruited from Physics, Chemistry, and Mathematics departments, and from departments in the College of Engineering. A snowball sampling procedure began with 21 randomly selected faculty from each department engaged in the STEM instruction of engineering students. These faculties then identified the colleagues they utilize as resources for improving their instruction. Characteristics of faculty connectedness in the network consisted of number of connections and depth of connections for both individual faculty and within and across departments. Outcome measures consisted of a survey of learner-centered attitudes and classroom observations of faculty instructional practices. The results show that faculty exhibiting more learner-centered behaviors had deeper and more extensive social networks than their peers who were more instructor-centered. The results are discussed in terms of the need for intra- and inter-departmental faculty professional development experiences that build and support collaborative organizations of faculty working together to transform early engineering experiences.
\end{abstract}

Keywords: faculty development; first-year programs; social network analysis; attitudes

\section{Introduction}

Recent research has shown that learner-centered practices are infrequently used in engineering pedagogy [1]. Learner-centered instruction is defined by the American Psychological Association [2] as comprising a set of cognitive and metacognitive factors, motivational and affective factors, developmental and social factors, and individual differences (see Table 1). It is assumed, generally, that to be learner-centered, pedagogies need to simultaneously address key concepts, skills, and dispositions in a domain, and account for the knowledge, predilections, and social characteristics afforded by students in a manner that is dynamic, adaptive, and personally relevant.

In a recent meta-analysis of 119 studies across grades K-20, [3] found that learnercentered variables such as non-directive verbal interactions, incorporation of higher-order thinking, encouraging learning and challenges, and adapting to individual and social differences correlate significantly with cognitive and affective student outcomes (e.g., mathematics achievement, science achievement, participation, motivation, and others). 
Relationships among these variables average $\mathrm{r}=0.34$, indicating that the overall influence of learner-centered practices accounts for about ten percent of desired outcomes-a significant relationship.

Table 1. American Psychological Association Learner-Centered Psychological Principles.

\section{Cognitive and Metacognitive Factors}

Nature of the learning process: The learning of complex subject matter is most effective when it is an intentional process of constructing meaning from information and experience.

Goals of the learning process: The successful learner, over time and with support and instructional guidance, can create meaningful, coherent representations of knowledge. Construction of knowledge: The successful learner can link new information with existing knowledge in meaningful ways.

Strategic thinking: The successful learner can create and use a repertoire of thinking and reasoning strategies to achieve complex learning goals.

Thinking about thinking: Higher-order strategies for selecting and monitoring mental operations facilitate creative and critical thinking.

Context of learning: Learning is influenced by environmental factors, including culture, technology, and instructional practices.

\section{Motivational and Affective Factors}

Motivational and emotional influences on learning: What and how much is learned is influenced by the learner's motivation. Motivation to learn, in turn, is influenced by the individual's emotional states, beliefs, interests, and goals, and habits of thinking. Intrinsic motivation to learn: The learner's creativity, higher order thinking, and natural curiosity all contribute to motivation to learn. Intrinsic motivation is stimulated by tasks of optimal novelty and difficulty, relevant to personal interests and providing for personal choice and control.

Effects of motivation on effort: Acquisition of complex knowledge and skills requires extended learner effort and guided practice. Without learners' motivation to learn, the willingness to exert this effort is unlikely without coercion.

\section{Developmental and Social Factors}

Developmental influence on learning: As individuals develop, they encounter different opportunities and experience different constraints for learning. Learning is most effective when differential development within and across physical, intellectual, emotional, and social domains is taken into account.

Social influences on learning: Learning is influenced by social interactions, interpersonal relations, and communication with others.

\section{Individual Differences Factors}

Individual differences in learning: Learners have different strategies, approaches, and capabilities for learning that are a function of prior experience and heredity.

Learning and diversity: Learning is most effective when differences in learners' linguistic, cultural, and social backgrounds are taken into account.

Standards and assessment: Setting appropriately high and challenging standards and assessing the learner and learning progress—including diagnostic, process, and outcome assessment-are integral parts of the learning process.

The hypothesis underlying the study reported here is that faculty practices are the primary mechanism by which potential changes in student learning and motivation are affected. Though faculty attitudes may support practical change, attitudes without implementation make no impact. Moreover, student beliefs and attitudes may mediate their learning behavior, but without practical change in the conditions of learning, counterproductive beliefs and attitudes have no means to change for the better. The research reported in this manuscript assumes that the conditions of learning are responsible for the nature and extent of learning, and students' beliefs about and self-regulatory strategies recruited while learning. Shifting faculty practices, therefore, is the primary driver of positive change in undergraduate engineering learning behaviors and outcomes. 
One of the problems in studying learner-centered practices and the conditions that occasion them is that they cast a fairly wide net. Many different pedagogical techniques may be employed effectively and be called "learner-centered." As such, learner-centered instruction can be thought of as heuristic-incorporating rules of thumb that can be used in the moment to tailor instruction to the needs of students. The following heuristics have been shown to be effective in structuring learner-centered pedagogical environments. As can be seen, learner-centeredness requires a shift from presenting content to enervating and supporting the actual learning modalities and outcomes for students.

1. The actions of the instructor focus on students learning as opposed to presenting material.

2. In ethically responsible ways, instructors share decision making about learning with students. Teachers control less, but students are involved more.

3. Content is used to build a knowledge base, to develop learning skills, and to foster learner self-awareness of their abilities. Teaching approaches account for students' learning modes and strategies.

4. Instructors and students, together, create learning environments that motivate students to accept responsibility for learning.

5. Assessment activities are used to promote learning and to develop self and peer assessment skills, not to evaluate performance primarily.

Unfortunately, research shows that learner-centered practices are used only infrequently by post-secondary science, technology, engineering, and mathematics (STEM) faculty [1]. Part of the problem concerns the fact that an instructor's perception of student-centered pedagogy may not align with the reality of their classroom practice. Ebert-May et al. [4], for example, reported on results of a national biology professional development program consisting of 6-12 days of workshops on scientific teaching over three years. While faculty engaged in professional development self-reported they were using a student-centered learning strategy, observation of their practice revealed that, in reality, three-fourths were lecturing with instructor-centered teaching. The authors maintain that the professional development was not sufficient to distinctly change the nature of their teaching practice. So, the majority of participants had not actually progressed into a true embodiment of learner-centered practice. Ebert-May et al. hypothesized that a possible reason for the lack of efficacy of the intervention was lack of social support for their changing beliefs, thus hindering their translation into new classroom routines and activities.

In support of this general hypothesis, McKenna et al. [5] studied engineering faculty developing student-centered conceptual change instructional methods. The faculty worked collaboratively with learning scientists to promote effective task and instructional sequence design. The researchers found that the greater the extent of collaborative reflection between engineering faculty and learning scientists, the greater the shift toward student-centered practices.

Other researchers [1] have used Everett Rogers' [6] model of diffusion of innovations to characterize faculty change through professional development. They found that faculty tend to only progress through the earliest stages of change, awareness and interest, and tend not to move to actual practice. The key issue here is that, without changes in students learning experiences, there is little chance of improving learning and motivational outcomes. Alternatively, if change occurs too slowly, it may be so ineffective that it may take years or decades for instructional practices to catch up with students' needs. Altogether, this paints a bleak picture of faculty practice and faculty development. However, Borrego et al. also cite convincing evidence that providing support in the form of collaborative interactions among faculty during the third and fourth decision and trial stages of can provide a successful progression to the higher stages of diffusion of innovation.

Though institutions tend to provide professional development opportunities, these are typically formal programs with expert content providers and facilitators. Most organizational learning, in contrast, happens through informal collaboration that exists among faculty. The problem for professional development is, though informal collaborations 
are more authentic and impactful than more formal professional development programs, they are mostly implicit, ad hoc, spontaneous, and invisible to others, and therefore are not available for targeted intervention [7]. The study reported in this article provides a methodology for examining these informal networks among faculty, and for assessing their potential impact on practice. Results from this study are being used to inform a professional development program that connects faculty more formally both within and across departments, with the hopes that stronger informal affiliations will develop, and that innovation in teaching will diffuse through these affiliations to impact the network of first-year engineering programs more broadly.

\subsection{Social Network Analysis}

Networks are important models for understanding both how to think about diverse organizations of human beings and to organize human beings into more optimal, collaboratively functioning systems [8]. This approach, rather than focusing on the individual attributes of group members, instead models the ways in which individuals share information one with another, the flow of that information, and the boundaries and limits of the information exchange.

Moolenaar characterizes the social network approach by three key principles that define it from other approaches to the sociology of education [9]. First, social resources, such as information, technologies, knowledge, and routines are exchanged in relationships among people. Individuals' social networks provide pathways for these resources to flow, and the propagation or sharing of resources across the network is accomplished through social interaction - in our case, asking a colleague for advice or meeting as a community of practice to discuss a potential way to improve student learning.

Second, a social network approach views the individual not as independent but interdependent with others in the larger social network. These interdependencies (i.e., relationships) exist at multiple levels in the system: for example, pairs of colleagues within program areas are embedded within departments. These higher-levels of the system, in turn, may be organized into cross-departmental structures such as research institutes or, in our case, first-year faculty. Importantly, change in lower-level collaboration is assumed to result in some change at higher levels in the social system [9].

Third, a social network approach assumes that the networks, as avenues or pathways for collaboration and resource exchange, provide both opportunities and barriers (through system inertia) to change in the larger system. Faculty may only work within the network, accessing information through the ties they currently have, or they may expend energy to create new connections and subnetworks that work around the inertia of the existing structure. This, of course, is easier at lower levels and more difficult at higher levels, wherein the relationships might be between departments or schools. Moolenaar maintains that, when the pattern of relationships for many faculty members is disconnected from the flow of resources in their unit, the ability of the unit or school at systematic improvement is hindered [9].

Yet, within any social network, certain individuals do exert influence across levels of the system. Such people are termed "bridges" or "brokers" if they span subgraphs in the larger network, allowing the flow of information across previously isolated groups. Social network analysis (SNA) enables brokers and other key players to be identified and the structure of the network to be empirically described and graphed visually and analyzed empirically.

Due to their central location in the network, brokers often have more access to best practices and, importantly, have the ability to communicate across the network, more so than faculty who are less central. One of the key promises of social network analysis is to be able to identify people who are more central or more peripheral to a network and help create ways to bring them closer to the core of the community. In intervention efforts such as the one described in this manuscript, social network analysis can measure communication 
flows and bottlenecks, which, in turn, may suggest interventions to enhance network function [8].

For example, researchers studied the degree of faculty connectedness in high school STEM teachers [10]. They recorded the degree to which teachers served as resources for others, in addition to seeking out others for assistance in improving their instruction. They found that the degree to which individuals served as sources of information, i.e., where others came to them for help, strongly predicted their implementation of constructivist pedagogies. In the current study, teaching practices assessed on the Reformed Teaching Observation Protocol are deemed close analogs to learner-centered practices, as defined by the APA and Weimar [11,12].

The research literature therefore suggests that social support for faculty is a key mediating variable determining faculty practice. There have been many reports of faculty, particularly adjuncts and teaching faculty, feeling unsupported and isolated in university STEM departments [13]. Where practical change occurs, research has shown that collaborative professional development can be one of the key design elements of the effort [4].

This study examines the extent of faculty social networks across university STEM departments and the relationship between faculty connectedness with their learner-centered attitudes and classroom practices. It is situated within a five-year faculty development project at a large public university in the Southwest United States. This project is using results from these analyses to stimulate communities of practice (CoPs) more effectively within and across departments to improve their teaching practices in first- and second-year engineering courses.

\subsection{Research Question}

The research question under investigation was: To what extent is the degree of social connectedness among faculty within and among STEM departments related to faculty attitudes about, and their degree of implementation of, learner-centered practices?

\section{Materials and Methods}

\subsection{Participants}

Participants were recruited from Physics, Chemistry, Mathematics, and Teacher Education departments, and from seven departments in the College of Engineering (Mechanical/Aerospace, Civil, Materials, Biological, Electrical, Computer, and Freshman Engineering) at a large, urban, Southwestern University in the United States. Sampling proceeded in two phases: (1) the sample began with 21 randomly selected faculty from each department engaged in the STEM instruction of first- and second-year engineering students (13 Engineering, 4 Physics, 2 Mathematics, and 2 Chemistry); (2) the second level consisted of 69 additional colleagues identified in an interview process by the first level participants as people they utilize as resources for improving their instruction. The faculty were provided small stipends as compensation for their time.

The 21 originally sampled were interviewed, observed, and surveyed in-depth regarding their attitudes and teaching practices. The remaining 69 were surveyed in the SNA portion of the study only.

\subsection{Interviews}

This study utilized four data collection methods: Faculty interviews, faculty surveys, observation protocol scores, and qualitative classroom observation.

Each faculty member in the study participated in one one-hour semi-structured interview focused on their practice and on the supports and barriers they encountered in their efforts to improve their practice. Interviews consisted of twenty-four questions focusing on topics of teaching practices, teaching resources used, teaching environment, course and departmental policies, self and departmental evaluations, and departmental and interdepartmental collaboration. Interviews were audio-recorded upon permission. The interviewer had no previous relationship with any of the interviewees. 
The Glaser and Laudel approach to qualitative content analysis was chosen to analyze the interview data [14]. Qualitative content analysis is a theory-guided method that extracts qualitative content using units of meaning originating from the same theoretical framework that guided the quantitative data collection. The goal of this analysis was to determine contextual and potentially causal factors in the qualitative data that "mirrors" the quantitative. Specifically, we used this method to ascertain the common resources faculty used to support instruction and then determine the extent to which use of resources supported learner-centered instruction as defined by the quantitative measures (below).

To obtain a larger sample for the SNA, a key portion of the interviews involved a set of questions focusing on the social network related to instruction within which each participant operated. Three questions were asked:

1. With whom do you collaborate regarding improvement of instruction?;

2. With whom do you discuss teaching-related topics?; and

3. Who comes to you to discuss instruction?

The interviewer probed instructors to create a comprehensive list of others, both within their department and outside their department. Responses also indicated that several faculty members rely on colleagues outside their university for information and support. A total of eighty-three persons were nominated using this process, across all participants. Outside nominations were discarded; one interviewee's quantitative data was incomplete and discarded, leaving 80 faculty in the remaining pool.

\subsection{Approaches to Teaching Inventory}

In addition to the interview, each of the 21 original instructors completed a 22-item revised edition of the Approaches to Teaching Inventory (ATI) survey to measure faculty perceptions about their own teaching. The ATI is a valid and reliable self-reporting tool designed by Trigwell and Prosser that assesses the extent to which faculty teach with an approach toward instructor-centered knowledge transmission versus student-centered conceptual change [15]. Items on the ATI fall into four dimensions: (1) conceptual change intention, measuring the degree to which instructors are aware of and support the development of student understanding in the class (e.g., I see teaching as helping students develop new ways of thinking in this subject); (2) student-centered strategies, measuring the extent to which instructors utilize pedagogical strategies that focus on student learning (teaching in this subject should help students question their own understanding of the subject matter); (3) information transmission, the extent to which the instructor emphasizes getting information to the student (e.g., I think an important reason for running teaching sessions in this subject is to give students a good set of notes); and (4) teacher-focused strategies (e.g., my teaching in this subject focuses on delivering what I know to the students). The first two dimensions promote student-centered classroom practice, while the latter two promote teacher-centered classroom practice. Reliabilities of the subscales range from $\alpha=0.73$ to 0.75. Of course, it was expected that all instructors will incorporate some beliefs from each of these four dimensions to more or less of a degree in their own teaching perspective.

\subsection{Observation Protocol and Observation Procedure}

For each course, at least three classroom observations were conducted. Six faculty had four observations, and two faculty were observed six times, for a total of 96 observations. Sections to be observed were randomly selected from the list of required freshman engineering courses taught in each department. The Reformed Teaching Observation Protocol (RTOP) was used after each observation to identify specific teaching practices associated with reformed teaching. The RTOP is a classroom observational protocol that quantitatively characterizes the extent to which faculty implement student-centered behaviors in their own classroom practice. It has high reliability and validity. Published reliabilities of RTOP subscales are: Lesson Design and Implementation $(\alpha=0.915)$, Propositional Knowledge $(\alpha=0.670)$, Procedural Knowledge $(\alpha=0.946)$, Communicative Interactions $(\alpha=0.907)$, and Student/Teacher Relationships $(\alpha=0.872)$. 
The overall RTOP has a reliability of $\alpha=0.954$. As classroom practice can vary across days and specific learning objectives, RTOP scores for each participant's three observations were averaged to gain a typical view of their practice, resulting in a single set of scale scores for each participant. In addition to RTOP scores, qualitative classroom observation field notes were gathered during each course observation, including details about class environment and student-instructor interactions. The classroom observation field notes were used to provide relevant information in conjunction with the RTOP results and to provide any needed context when examining the relationship between the classroom teaching practices, as reflected in the RTOP score, and the teaching beliefs reflected in the instructor interviews and survey responses [12].

\subsection{Social Network Analysis}

Social network analysis is a set of analysis techniques that characterize the number and type of connections among members of a social system. It uses mathematical concepts from graph theory to map the links (relationships) between nodes (people). Any adjacency matrix listing the connections among individuals can be turned into a vertex-edge graph, which illustrates visually the density of connections within and cross-connections across departments. We followed this method in another earlier study [16] fruitfully to predict the relationship between high school STEM teacher connectedness and dimensions of the RTOP. Specifically, we study indegree, the extent to which colleagues approach a participant to discuss teaching practices, and outdegree, the extent to which participants seek out another to assist in their own teaching practice.

Here, we provide more depth into the process used to develop participant's social networks related to instruction. We used a method termed snowball sampling, in which an original set of faculty members teaching first-year courses were randomly selected, stratified by departments. This original set of 21 were then interviewed to determine whom they "go to" for information about improving instruction, and who "comes to them" for help about improving instruction. The faculty nominated by this original sample were then surveyed to determine whom they go to and who comes to them. This process yielded a final set of 80 participants for the SNA.

Names of the 80 identified faculty were put into columns in an online survey matrix. Each participant was asked to check a box indicating whether they "go to" another colleague to share teaching strategies, and then asked to check a box whether each faculty member "comes to" them to share teaching strategies. Faculty responses were then arranged into an adjacency matrix showing indegree and outdegree. These responses are displayed using a directed graph (digraph) to show the directionality of influence for each faculty member. The digraph is drawn, placing faculty with the most common connections in proximity, and organizing them by departmental affiliation to more clearly illustrate within- and cross-departmental connections.

\section{Results}

The adjacency matrix for all 80 participants was analyzed using Matlab 1015b biograph function. Figure 1 shows the connectedness among all participants. Both the density of connections within departments and the connectedness across departments are of importance here. It is clear from the area comprised by each department that there is much more collaboration in-department than across-departments. It is also clear from the density of connections in each department that the interconnections among members of Bioengineering, Physics, and Freshman Engineering is higher than the other departments. These departments contain many participants involved in the design and implementation of first-year instruction for engineering students, they have cultures of collaboration and, importantly, they have histories of projects and funding that support faculty collaboration for the purpose of improving instruction. It is also apparent is that there are few connections across departments, with notable exceptions from the Freshman Engineering Department, whose faculty hold positions concurrently with disciplinary departments. 


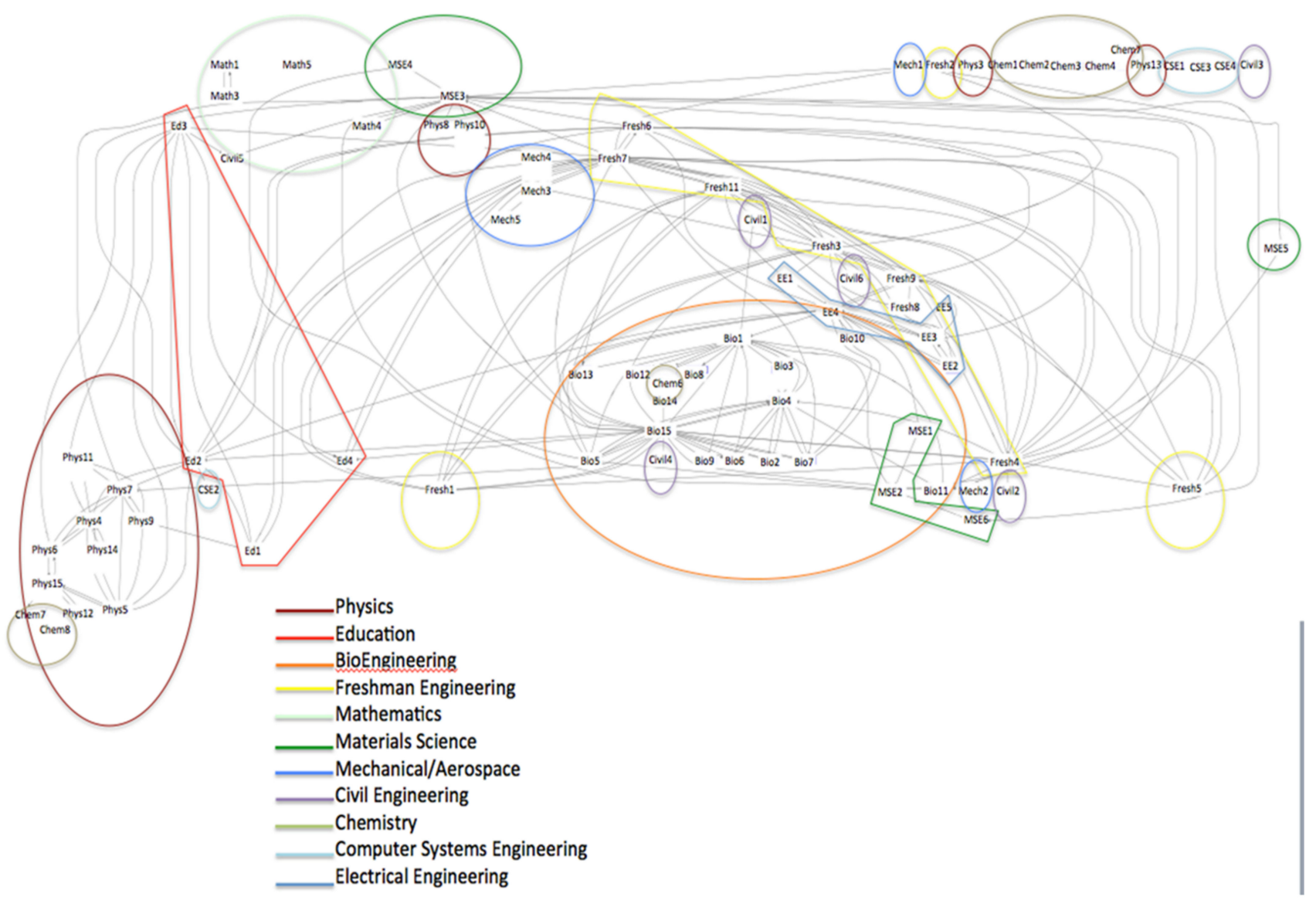

Figure 1. Directed graph of faculty connections organized by departmental affiliation.

\subsection{Indegree and Outdegree}

The mean indegree for faculty across the network was 1.5 connections (SD =1.4), and the mean outdegree was 2.3 connections $(S D=2.1)$. This implies that, on average, the faculty surveyed were assisted by two or three with their teaching and, in turn, provided assistance to one or two, but there is high variability. Over one-third of the sample had no colleagues in the social network with whom they interacted for the purposes of improving instruction, but some (BIO15 and FRESH3, for example) have many connections-their indegree is especially high (five each). These two faculty members serve, in their respective departments, as instructional leaders, sharing practices and materials with their colleagues freely.

Degree estimates in this analysis are likely low, since we did not sample the entire set of colleague connections across faculty serving engineering students in their first two years at the university. Nevertheless, it does indicate that each individual's real contact for their own professional development is quite limited, given the university employs over 700 faculty in the surveyed departments. Table 2 presents the mean out-degree and indegree of each department.

Table 2. Mean Outdegree and Indegree Across Participating Faculty Departments.

\begin{tabular}{ccccccccccccc}
\hline & Bio & Chem & Civil & Comp & Ed & Elect & Fresh & Math & Mech Aero & Mater & Phys & Overall \\
\hline Out deg. & 2.6 & 0.71 & 1.0 & 0.25 & 2.75 & 2.4 & 5.4 & 1.5 & 1 & 3.33 & 1.85 & 2.3 \\
In deg. & 1.6 & 0.57 & 0.5 & 0.00 & 1.25 & 1.4 & 3.3 & 0.25 & 1 & 2.17 & 1.38 & 1.5 \\
\hline
\end{tabular}

\subsection{Depth of Faculty Network}

Figure 2 presents the minimal spanning tree for the faculty network. A minimal spanning tree is a graph that connects all the nodes together, accounting for the direction 
of flow, in our case, the direction of faculty influence, with a minimum number of edges. A quick look shows that, at most, the depth of the tree, beginning at any single faculty member, is at most five (beginning at MECH4, a Mechanical/Aerospace faculty member) and averages between one and two colleagues. This means that, for information about new teaching practices, technologies, or other innovations to spread across the network as a whole, it must propagate through at least five intermediaries and their connections and, for most faculty, many more.

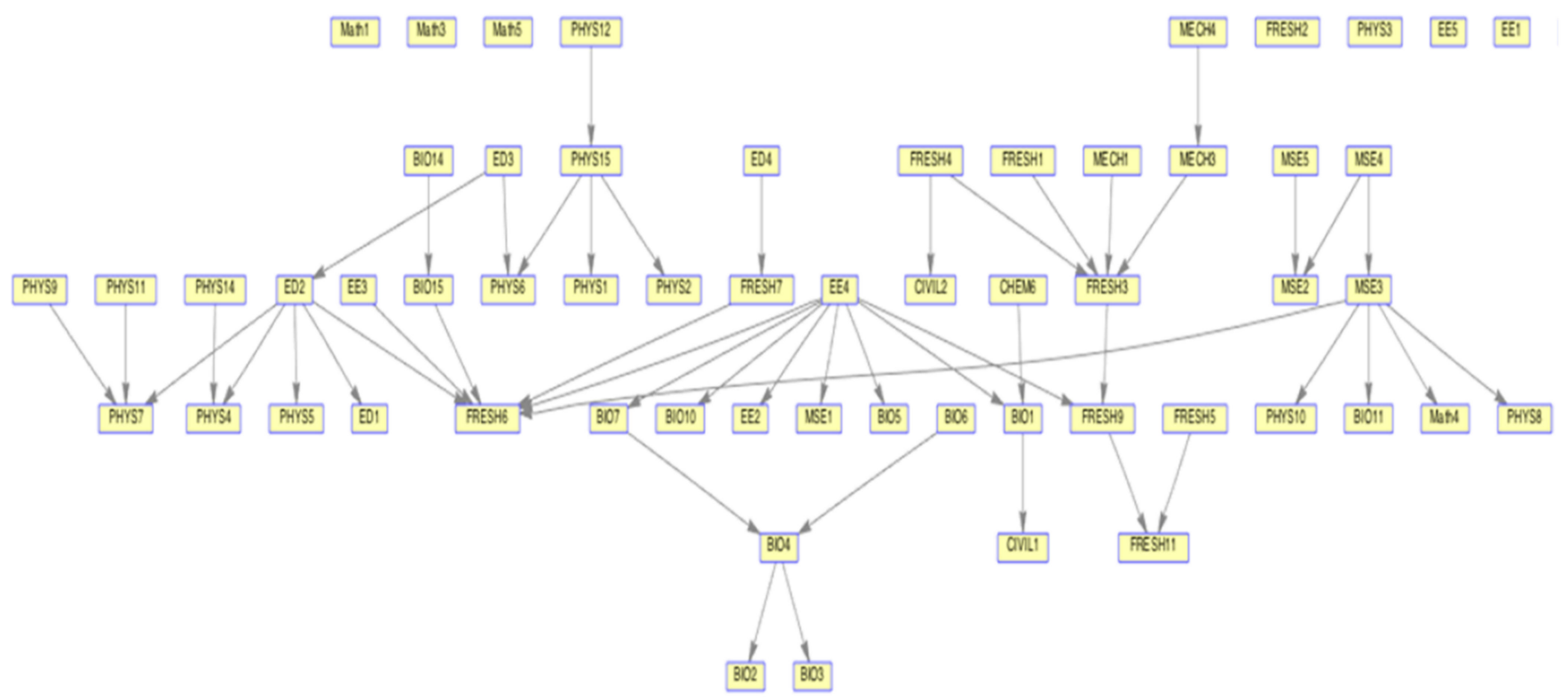

Figure 2. Minimal Spanning Tree of the Faculty Social Network.

This shallow depth, coupled with the fact that 29 of 80 participants could not be connected in the tree illustrate that it is impossible to reach all faculty members given current "natural" relationships. Some means of providing institutional pressure or support to connect faculty across departments is critical if instruction is to improve in an expanding community of practice. Our current project aims to provide appropriate stipends and support of teaching through observation and feedback to incentivize the establishment of larger communities of practice within departments.

Thus far, we have established that first-year engineering faculty in our university, by and large, do not have extensive communication with each other related to the improvement of their instructional practice. We now show that such connections among faculty are related to the degree to which participants engage in learner-centered teaching practices.

\subsection{Relationship between Faculty Connectedness and Learner-Centered Attitudes and Practice}

To test the hypothesis that more connected faculty, on average, engage in more learnercentered practices, we computed Pearson correlations between scores on the ATI beliefs instrument and the RTOP observational instrument, and the degree of connectivity exhibited by each participant (see Table 3). 
Table 3. Pearson correlations between degree of connectedness and dimensions of faculty learnercentered attitudes (ATI) and classroom practice (RTOP).

\begin{tabular}{cccccccccccccc}
\hline \multicolumn{1}{c}{ ATI Dimensions } & \multicolumn{4}{c}{ RTOP Dimensions } \\
\hline & $\mathbf{1}$ & $\mathbf{2}$ & $\mathbf{3}$ & $\mathbf{4}$ & $\mathbf{5}$ & $\mathbf{6}$ & $\mathbf{7}$ & $\mathbf{8}$ & $\mathbf{9}$ & $\mathbf{1 0}$ & $\mathbf{1 1}$ & $\mathbf{1 2}$ \\
\hline Out deg. & 0.059 & 0.270 & -0.351 & -0.290 & 0.212 & -0.367 & $0.528^{*}$ & 0.020 & $0.453^{*}$ & 0.347 & 0.277 & 0.420 \\
\hline In deg. & 0.277 & 0.395 & -0.140 & -0.106 & 0.400 & -0.142 & $0.617^{* *}$ & 0.053 & $0.555^{*}$ & 0.393 & 0.408 & $0.490 *$ \\
\hline
\end{tabular}

Note: ATI Dimensions: 1. Conceptual change intention, 2. Student-focused strategies, 3. Information transmission, 4. Teacher-focused strategies, 5 . Conceptual change/student focus composite, 6 . Information transmission/teacher focus composite. RTOP Dimensions: 7. Lesson design and implementation, 8. Propositional knowledge, 9. Procedural knowledge, 10. Communicative interactions, 11. Student/teacher relationship, and 12. RTOP composite. ${ }^{*} p<0.05 . * * p 0.01$

Overall, correlations are low to moderate across most dimensions of faculty learnercentered beliefs and classroom practice. The directions of relationships are all as expected, indicating a positive relationship between connectedness and learner-centeredness in general, and a negative relationship between connectedness and teacher-centeredness. RTOP scores were generally very strongly related to connectedness, with lesson design and implementation showing the strongest relationship. This dimension focuses on strategies that account for students' prior knowledge, the engagement of students as members of a learning community, the encouragement of alternative modes of investigation and problem solving and using students' ideas to help direct the lesson. The second highest correlation centered on procedural knowledge, a dimension emphasizing rigor of ideas, multiple representations, and engagement in hypothesis building and testing. The least correlation centered on propositional knowledge. This dimension focused on the subject matter knowledge of the teacher, the abstractness of the content, connections to other disciplines, and the coherence of the conceptual understanding promoted by the lesson.

It is also important to note that none of the ATI dimensions were significantly correlated with connectedness. While technically, this indicates no systematic relation between the variables, the relatively small number of participants in the study yields relatively low power. With an earlier study [16] showing significant positive relationships between ATI and RTOP scales, we feel there is still merit in reporting these correlations as consistent with the theory. However, it is the overall pattern of the relationship, not each individual correlation, that should be examined.

\section{Discussion}

In the context of large-scale reform of the first two years' STEM teaching practice at a major research University, the present study shows that faculty isolation is a quantifiable phenomenon. Moreover, the quantity of instructors' interconnectedness related to sharing teaching practices is significantly related to their knowledge of, and ability to, implement learner-centered practices in their beginning courses for engineering majors. Relatively strong correlations to learner-centered practices are especially important, since those practices are the key to change in the experiences of freshman and sophomore students, potentially improve their learning and understanding of foundational concepts and skills and their enjoyment and satisfaction with these courses as indicative of their future success in their engineering major. This latter supposition, of course, was not tested in the present study. This is necessary future work.

Our findings are supported in a recent paper, in which the authors surveyed STEM faculty to ascertain the relationship between adoption of evidence-based instructional practices and their communities of practice [17]. Social network analysis examined both conversation communities, in which faculty interacted with each other on any topic, versus collaboration communities, in which they interacted specifically around understanding and implementing evidence-based instructional practices (EBIP) —a set of practices that is functionally equivalent to our RTOP assessment. Like our study, the authors found that faculty teaching networks tend to center around departmental affiliation. Faculty 
communities adopting evidence-based practices-practices that we would consider learnercentered-would often utilize a central person or team to facilitate communication and collaboration. However, members tended to feel free to start collaborating with anyone in the EBIP community. For non-adopters, the reliance on a central person or team was also found, but this reliance was more pronounced-many fewer collaborations existed across non-central members.

In other words, evidence-based, learner-centered teaching practices appear to be highly related to the degree to which instructors are seen as resources by their colleagues. Trigwell and Prosser [15] suggest that, in a social network, the degree to which an individual is seen as a resource, where their colleagues come to them for assistance, is an indication of their leadership.

Moreover, like Spillane's study of literacy and mathematics teachers in the K12 setting, we found that work-related interactions among faculty depend on the subject matterwitness the much higher number of within-department connections as opposed to crossdepartment connections-even in the current study, where all content was ostensibly STEM related [18].

The number of people in a network with large indegree indices is a good measure of closeness of collaboration. Friedkin and Slater suggest that such sources of advice and creativity can be more influential in the social network than more formal leadership roles [17]. Ma et al. found that, in their study of engineering departments, network density indicated the degree of functionality of faculty communities of practice. In their study, dysfunctional CoPs showed fewer within-community connections than functional CoPs. Similar to the present study, Ma et al. found that more functional communities had more cross-community connections with other functional communities. Less-functional communities rarely showed cross-community collaboration [19].

We also found this to be the case with Bioengineering, Freshman Engineering, and Physics especially, having closer within-department relationships and significantly greater collaboration across departments. Moreover, these departments show extensive support in terms of funded projects, professional development, team meetings, and official repositories of faculty teaching materials, by which their intra-departmental teaching culture can flourish. Mathematics, Chemistry, and Computer Systems Engineering showed markedly fewer intra-departmental connections and markedly less explicit support for improvement of instruction.

The sparseness of the minimal spanning tree for the network is additionally concerning: current faculty relationships, being primarily intra-departmental and shallow (averaging fewer than 2 degrees of separation), prevent large-scale propagation of teaching innovations without significant investment in forging inter-departmental collaboration. The few faculty members with large indegree might serve, in this case, as important brokers of innovation. As acknowledged leaders in their informal in-department network, they might be recruited as ideal candidates for sharing their innovations across departments, given administrative and fiscal support. If the findings from each of these studies are generalizable, then there is reasonable evidence to assume that within-unit connections are requisite for healthy cross-unit collaboration.

Brokers, such as those in our study with large indegree, are largely considered critical for the diffusion of innovation, as they provide validation of the efficacy of new strategies independent of the school's administration or the faculty champions who may lead professional development. In essence, such brokers represent proofs, in the eyes of participants, that the innovation can be successful, and that their experiences can be used as models for the implementation of practices that they found successful (e.g., being individuals that serve a similar role as innovation brokers in institutional networks [8]).

\section{Conclusions}

In summary, the research reported in this paper provides further evidence that faculty culture is moderately to strongly related to quality practice-at least as a mediating variable 
for learner-centered instruction. Faculty with more connected social networks displayed higher scores on conceptual change and student-focused attitudes and lower scores on information transmission and teacher-focused attitudes than faculty with fewer connections. Moreover, faculty with larger social networks scored highly on all learner-centered variables, with the exception of the propositional knowledge subscale on the RTOP. None of the correlations for faculty attitudes were statistically significant. However, the strong correlations for faculty practice variables lends support for our original hypothesis.

Regarding limitations, a first consideration is that of generalizability. The patterns of relationships found in this study may not reflect the patterns at another university with different departmental structures, support for teaching, and faculty roles and responsibilities. Nevertheless, it does provide some indication that natural affiliations among faculty may not be adequate for the effective diffusion of innovation in teaching practices at scale, and that there is a likelihood for interdepartmental isolation of faculty teaching in the first two years of the curriculum. The unique SNA picture drawn was also no doubt influenced by the random selection of the initial faculty members teaching first-year courses. Though it is contended that the overall structure and correlation patterns would persist with repeated random selection of faculty members and replicating the study, subtle and perhaps moderate differences might be observed with each iteration. Further examination of faculty networks and diffusion of innovation should be examined across college type, department size, and discipline, which often has very distinct cultural characteristics.

The results of this study are consistent with recent work using social network analysis to explain potential ways in which the organization of faculty for professional development and collaborative improvement of instruction can be positively impacted. Specifically, this body of work suggests that faculty with a high indegree may be necessary catalysts to the development and growth of communities of practice that are more collaborative with respect to sharing and adopting best practices for teaching. Additionally, it may also be that over-reliance on these central faculty for faculty with high out-degree may cause bottlenecks that prevent propagation of best practices across the community.

A further limitation to consider is that we did not examine the specific kinds of resources shared across faculty networks. It is likely that sharing materials versus engaging in meaningful discourse about instructional practices would have dramatically different impact on learner-centered teaching. When designing communities of practice for faculty development, research suggests that networks will be more efficient and effective when colleagues are aware of each other's expertise, when they have adequate access to their colleagues' expertise, when the larger structure of the network is perceived as a safe environment for learning and trying out innovations, and when there is a commitment from members of the network to the active exchange of resources. Further, we did not examine the collaboration between faculty on research or teaching projects in great detail, which could further illuminate exchange of information and development of faculty networks. The design of professional development programs should be based, to a large extent, on these conditions to capitalize on the potential of faculty social networks for the improvement of practice [9]. The extent to which natural brokers-those with large indegree, suggesting that they have acknowledged expertise among their peers-can be recruited to strengthen faculty connections within and across departments is a potentially powerful testable hypothesis for the improvement of instruction in the first two years of an engineering curriculum.

A final limitation recognized is that data for this study were collected prior to the COVID-19 pandemic. Research indicates the pandemic has considerably affected facultyto-student and faculty-to-faculty interactions $[20,21]$. Therefore, interpretations regarding how faculty interact with one another, as well as relationships between beliefs and practices, must be considered through our current and potentially enduring COVID-influenced lens. Therefore, similar studies are encouraged to assess the ways in which faculty beliefs, practices, and interactions have adjusted, and to determine how place-based university social networks persist or change and how they are influenced by burgeoning outside resources. 


\begin{abstract}
Author Contributions: Conceptualization, J.A.M., S.K. and E.J.; Data curation, L.R. and K.L.H.; Formal analysis, J.A.M. and L.R.; Funding acquisition, J.A.M. and S.K.; Investigation, K.L.H.; Methodology, J.A.M., E.J. and K.L.H.; Project administration, S.K.; Writing—original draft, J.A.M.; Writingreview and editing, S.K., E.J., L.R., R.C., K.D.H., K.L.H. and Y.-C.C. All authors have read and agreed to the published version of the manuscript.
\end{abstract}

Funding: This material is based upon work supported by the National Science Foundation under Grant No. (1226586).

Institutional Review Board Statement: All subjects gave their informed consent for inclusion before they participated in the study. The study was conducted in accordance with the Declaration of Helsinki, and the protocol was approved by the Institutional Review Board of Arizona State University, under the Authority of the Arizona Board of Regents (Study \#1306009354).

Informed Consent Statement: Written informed consent has been obtained from the subjects to publish this paper.

Data Availability Statement: The data presented in this study are available on request from the corresponding author. The data are not publicly available due to confidentiality of participants and departments.

Conflicts of Interest: The authors declare no conflict of interest.

\title{
References
}

1. Borrego, M.; Froyd, J.E.; Hall, T.S. Diffusion of engineering education innovations: A survey of awareness and adoption rates in US engineering departments. J. Eng. Educ. 2010, 99, 185-207. [CrossRef]

2. American Psychological Association. Learner-Centered Psychological Principles: A Framework for School Redesign and Reform; ERIC Clearinghouse: Washington, DC, USA, 1995.

3. Cornelius-White, J. Learner-centered teacher-student relationships are effective: A meta-analysis. Rev. Educ. Res. 2007, 77, 113-143. [CrossRef]

4. $\quad$ Ebert-May, D.; Derting, T.L.; Hodder, J.; Momsen, J.L.; Long, T.M.; Jardeleza, S.E. What we say is not what we do: Effective evaluation of faculty professional development programs. BioScience 2011, 61, 550-558. [CrossRef]

5. McKenna, A.F.; Yalvac, B.; Light, G.J. The role of collaborative reflection on shaping engineering faculty teaching approaches. J. Eng. Educ. 2009, 98, 17-26. [CrossRef]

6. $\quad$ Rogers, E.M. Diffusion of Innovations; Free Press: New York, NY, USA, 2003; Volume 5.

7. Cross, R.; Parker, A. Charged up: Creating energy in organizations. J. Organ. Excell. 2004, 23, 3-14. [CrossRef]

8. Kezar, A. Higher education change and social networks: A review of research. J. Higher Educ. 2014, 85, 91-125. [CrossRef]

9. Moolenaar, N.M. A social network perspective on teacher collaboration in schools: Theory, methodology, and applications. Am. J. Educ. 2012, 119, 7-39. [CrossRef]

10. Judson, E.; Lawson, A.E. What is the role of constructivist teachers within faculty communication networks? J. Res. In Sci. Tch. 2007, 44, 490-505. [CrossRef]

11. Weimer, M. Learner-Centered Teaching: Five Key Changes to Practice; John Wiley \& Sons: San Francisco, CA, USA, 2002.

12. Sawada, D.; Piburn, M.D.; Judson, E.; Turley, J.; Falconer, K.; Benford, R.; Bloom, I. Measuring reform practices in science and mathematics classrooms: The reformed teaching observation protocol. Sch. Sci. Math. 2002, 102, 245-253. [CrossRef]

13. Dolan, V. The isolation of online adjunct faculty and its impact on their performance. Int. Rev. Res. Open Distance Learn. 2011, 12, 62-77. [CrossRef]

14. Glaser, J.; Grit, L. Life with and without coding: Two methods for early-stage data analysis in qualitative research aiming at causal explanations. Forum Qual. Sozialforsch. 2011, 14. [CrossRef]

15. Trigwell, K.; Prosser, M. Development and use of the approaches to teaching inventory. Educ. Psychol. Rev. 2004, 16, 409-424. [CrossRef]

16. Middleton, J.A.; Judson, E.; Krause, S.J.; Culbertson, R.J.; Mayled, L.H.; Ross, L.; Hjelmstad, K.L.; Hjelmstad, K.D.; Chen, Y.C. Social Network Analysis of Faculty Connections in a Multi-year Professional Development Program. In Proceedings of the ASEE Annual Conference \& Exposition, Salt Lake City, UT, USA, 24-27 June 2018.

17. Friedkin, N.E.; Slater, M.R. School leadership and performance: A social network approach. Sociol. Educ. 1994, 67, 139-157. [CrossRef]

18. Spillane, J.P. Primary school leadership practice: How the subject matters. Sch. Leadersh. Manag. 2005, 25, 383-397. [CrossRef]

19. Ma, S.; Herman, G.L.; West, M.; Tomkin, J.; Mestre, J. Studying STEM faculty communities of practice through social network analysis. J. High. Educ. 2019, 90, 773-799. [CrossRef]

20. Rupnow, R.; LaDue, N.D.; James, N.M.; Bergan-Roller, H.E. A perturbed system: How tenured faculty responded to the COVID-19 shift to remote instruction. J. Chem. Educ. 2020, 97, 2397-2407. [CrossRef] 
21. El Said, G.R. How did the COVID-19 pandemic affect higher education learning experience? An empirical investigation of learners' academic performance at a university in a developing country. Adv. Hum.-Comput. Interact. 2021, 2021, 1-10. [CrossRef] 